\title{
Eurostudia
}

\section{La sociologie, mise en abîme}

Essai avec la participation non autorisée de Milan Kundera

\section{Sociology as Mise en Abyme: An Essay with the Non-Authorized Participation of Milan Kundera}

\section{Barbara Thériault}

Volume 9, numéro 1, 2014

Lire Musil en sociologue

Reading Musil as Sociologist

Musil lesen als Soziologe

URI : https://id.erudit.org/iderudit/1026719ar

DOI : https://doi.org/10.7202/1026719ar

Aller au sommaire du numéro

Éditeur(s)

Le Centre canadien d'études allemandes et européennes

ISSN

1718-8946 (numérique)

Découvrir la revue

Citer cet article

Thériault, B. (2014). La sociologie, mise en abîme : essai avec la participation non autorisée de Milan Kundera. Eurostudia, 9(1), 41-50.

https://doi.org/10.7202/1026719ar
Résumé de l'article

À partir d'un exemple tiré de l'oeuvre du romancier contemporain Milan Kundera, je tente dans ce bref essai de délinéer une qualité de la sociologie et du travail de sociologue. Si je choisis ce romancier, c'est en raison d'une expérimentation à laquelle il se livre : il met en scène un personnage que j’affectionne particulièrement, Monsieur Engelbert. Cet « ego expérimental » m'accompagne dans mon enseignement et c'est avec lui que je pense les contours d'une perspective sociologique. Je suis également attentive à son contexte, le premier quart du $20^{\mathrm{e}}$ siècle en Europe centrale, et réfléchis à la forme que Kundera lui fait emprunter : l'autobiographie.
Tous droits réservés $@$ Le Centre canadien d'études allemandes et européennes, 2014
Ce document est protégé par la loi sur le droit d'auteur. L'utilisation des services d’Érudit (y compris la reproduction) est assujettie à sa politique d'utilisation que vous pouvez consulter en ligne.

https://apropos.erudit.org/fr/usagers/politique-dutilisation/ 


\title{
Barbara Thériault
}

Université de Montréal

\section{Résumé :}

À partir d'un exemple tiré de l'œuvre du romancier contemporain Milan Kundera, je tente dans ce bref essai de délinéer une qualité de la sociologie et du travail de sociologue. Si je choisis ce romancier, c'est en raison d'une expérimentation à laquelle il se livre: il met en scène un personnage que j'affectionne particulièrement, Monsieur Engelbert. Cet "ego expérimental " m'accompagne dans mon enseignement et c'est avec lui que je pense les contours d'une perspective sociologique. Je suis également attentive à son contexte, le premier quart du $20^{e}$ siècle en Europe centrale, et réfléchis à la forme que Kundera lui fait emprunter : l'autobiographie.

\section{Sociology as Mise en Abyme: An Essay With the Non-Authorized PARTICIPATION OF Milan KUNDERA}

\begin{abstract}
:
Drawing on an example from one of Milan Kundera's essays on the novel, I explore in this brief essay the qualities of the sociological approach and of the sociologist's work. I chose this novelist because of his experimentations with a character that I am particularly fond of, Mister Engelbert. His "experimental ego" accompanies me while teaching; and I often think about him when delineating the contours of a sociological perspective. I am also attentive to his context, the first quarter of the $20^{\text {th }}$ century in central Europe, and reflect on the form Kundera makes him use: the autobiography.
\end{abstract}

Serait-ce un aveu ? Les romanciers m'ont souvent aidée à comprendre ce que les sociologues parviennent à faire. Leur influence n'est pas étrangère à mon choix de devenir moi-même sociologue, et je n'hésite jamais à recourir à eux pour me seconder dans mon enseignement. En empruntant les chemins tracés par les traditions qui leur sont propres, les observateurs que sont ces écrivains en viennent souvent à partager une démarche rejoignant les objectifs de la 
sociologie: rendre visible ce qui est devant nous, mais que nous ne remarquons pas toujours.

Ma compréhension de la sociologie est celle d'une discipline qui nous demande de penser par objets, un peu à la manière du romancier. Bien qu'un tel parallèle puisse surprendre, il rejoint une perspective partagée par plusieurs de mes collègues sociologues et permet d'articuler une interrogation sur les formes et l'écriture sociologique qu'ils ne sauraient aujourd'hui contourner.

À partir d'un exemple tiré de l'œuvre du romancier contemporain Milan Kundera, je traiterai dans ces quelques pages de la sociologie et du travail de sociologue. Si je choisis ce romancier, c'est en raison d'une expérimentation à laquelle il se livre en mettant en scène un personnage que j'affectionne particulièrement, Monsieur Engelbert. Cet «ego expérimental » m'accompagne dans mon enseignement et c'est avec lui que je pense les contours d'une perspective sociologique. Je suis également attentive à son contexte, les années 1920 en Europe centrale, et réfléchis à la forme que Kundera lui fait emprunter : l'autobiographie.

\section{Un roman}

\section{Monsieur Engelbert}

Dans son dernier livre sur l'art du roman, Milan Kundera (Le rideau, 2005 : 146150) décrit une scène : debout devant le bibliothèque d'un ami qu'il visite à Prague, il en tire un livre. Intitulé Le Monstre à explosion, il s'agit d'un roman paru vers 1932 dans lequel Jaromir John, un auteur tchèque qui nous serait resté inconnu s'il n'avait été de Kundera, y dépeint l'histoire d'un homme, Monsieur Engelbert.

Monsieur Engelbert vit à Prague, au début de la République tchécoslovaque, dans le premier quart du $20^{\mathrm{e}}$ siècle. La République a été proclamée en 1918 après l'effondrement de l'Empire austro-hongrois. Kundera ne donne que peu de détails sur le personnage; on apprend qu'il est un fonctionnaire à la retraite de l'ancien régime et qu'il est un homme grave, correct. L'épisode de sa vie qui est relatée dans le roman se déroule dans les années 1920, une période marquée par des transformations particulièrement rapides et profondes. Des événements, d'abord, de nature politique : en 1918, à la fin de la Première Guerre mondiale, un nouvel État voit le jour. Prague est 
sa capitale et on y met en place une démocratie parlementaire dont les dirigeants sont élus au suffrage universel. Cette période coïncide également avec des changements aux plans économique et social. Les contemporains de Monsieur Engelbert sont témoins de la rapide industrialisation du jeune pays : l'économie capitaliste prend un essor remarquable, elle créée de la richesse, mais aussi de nouvelles formes d'inégalité dont la population est de plus en plus consciente. Kundera ne relate pas ces événements; ils sont en arrièreplan $^{1}$. Ces transformations n'en sont pas moins fondamentales: c'est un monde qui disparaît au profit d'un autre. De nouveaux acteurs entrent en scène, des élites issues de ce que les classes jusque-là dirigeantes appelaient, avec un serrement dans la gorge, les «masses ». Ces dernières ont des partis qui leur sont propres, socialistes et socio-démocrates dont les élus siègent au parlement. Jusque-là, les hommes issus des classes non-possédantes n'avaient accès ni au vote, ni à des postes politiques.

Tous ces changements nous paraissent marquants et, bien que l'histoire ne les avait alors pas encore ordonnés avec clarté, ils l'étaient aussi pour des hommes comme le protagoniste du Monstre à explosion. J'imagine Engelbert en homme intéressé par la chose publique, lecteur attentif des journaux. Je l'imagine au café - on est quand même à Prague! - , discutant de tous ces bouleversements. Je me le représente dédaignant cette forme encore nouvelle et populaire qu'est le cinéma, mais ne pouvant songer vivre sans certains autres développements technologiques passés comme les chemins de fer qu'il emprunte régulièrement, l'éclairage au gaz de sa rue ou son bain chaud. Dans son quotidien, Engelbert a de petits tracas, des soucis. Aussi petits soient-ils, ils occupent néanmoins une grande partie de son temps. Engelbert est surtout ennuyé par une chose: le bruit, le bruit des moteurs de ces voitures et motocyclettes qui envahissent peu à peu sa ville - ces nouveaux engins dont Le Monstre à explosion tire d'ailleurs son titre. Monsieur Engelbert est décrit comme un homme ennuyé, énervé, voire traqué : il ne parvient pas à échapper au bruit des moteurs. Tout ce qu'il veut, c'est dormir. Et il met en œuvre

\footnotetext{
${ }^{1}$ Dans ses romans, Kundera décrit peu ses personnages et les événements historiques. Ce sont les problèmes existentiels, les possibilités humaines, qui retiennent son attention (voir Kundera 1986: 46 et suiv.). Il nous laisse le soin de les imaginer. Il souhaite, je présume, éviter que nous tirions des conclusions trop hâtives de détails biographiques. Je vois quant à moi Monsieur Engelbert portant une moustache soignée et un costume de laine. J'ai envie de connaître des détails qui renvoie à des questions qui nous préoccupent aujourd'hui. Par exemple : comment s'appelait-il ? Karl ? Ou encore Karel ? Quelles langues parlaient-il ? Et dans quels circonstances et contextes les parlaient-ils?
} 
plusieurs stratégies pour arriver à cette fin : il déménage dans un quartier résidentiel, séjourne dans une ville de province, passe du temps à la campagne; rien n'y fait. Après de nombreux efforts, il finit par trouver son refuge : les trains de nuit, dont le balancement doux et régulier lui permettent enfin de retrouver le sommeil perdu.

\section{L'expérimentation : le personnage sort des pages du roman}

Après avoir raconté l'histoire et nous l'avoir laissé imaginer, Kundera se livre à une petite expérimentation qui n'est pas étrangère au travail du sociologue. Il s'imagine que le fonctionnaire à la retraite sort des pages du roman et qu'il est là, vivant, devant lui.

Kundera voit Engelbert - et nous le voyons avec lui — assis à sa table de travail. Engelbert semble concentré. Que fait-il ? Il écrit son autobiographie. Dans ses écrits, il réfléchit aux grandes questions de son temps: «(..) l'exploitation, l'inégalité, oui, cent fois oui, ce sont là des notions graves, capables de donner du sens à un destin, de rendre noble un malheur! » (Kundera $2005: 150$ ).

Kundera voit encore quelque chose, un détail. Assis à sa table de travail, le corps penché, Engelbert est en train d'écrire son autobiographie et il a quelque chose dans les oreilles. N'est-ce pas du coton ? Si, il a du coton dans les oreilles pour écrire en toute quiétude. Le coton n'est pas un thème de l'autobiographie d'Engelbert. En fait, il est fort à parier qu'il trouverait étrange que l'on s'y attarde et qu'il serait un peu gêné d'être ainsi surpris dans son intimité (Kundera 2005 : 150). Ce détail est néanmoins important. En effet, le thème du bruit auquel il renvoie, ce bruit auquel Engelbert cherche désespérément à échapper, est lié aux problèmes de l'heure, aux développements industriels et technologiques et à leurs conséquences quotidiennes et banales, des conséquences à première vue individuelles mais qui, au final, affectent tout le monde.

\section{$2 \mathrm{Du}$ roman à la sociologie}

L'histoire d'Engelbert racontée par Kundera est un bel exemple d'un observateur - qu'il soit romancier ou sociologue - qui parvient à télescoper de petites choses quotidiennes à de grands événements, à lier la vie d'un individu à celle de ses contemporains. Elle correspond à ce qu'un sociologue du nom de Charles Wright Mills appelait «l'imagination sociologique». Si 
cette qualité n'est pas l'apanage des sociologues, elle est néanmoins constitutive de leur travail. Elle renvoie à la capacité de comprendre le rapport complexe entre leurs épreuves personnelles et les bouleversements de l'histoire (Mills 1959 [1967] : 10).

Que fait l'observateur qui a développé cette qualité ? Il recadre la réalité et, ainsi, l'expose sous un autre jour. Pour le romancier, tout comme pour le sociologue, cette qualité d'esprit passe par une attention soutenue aux détails². Elle peut être aiguisée par des jeux d'échelles, de mises à distance et de rapprochements, des changements de perspectives (Mills 1959 [1967] : 11) — en passant du plus grand au plus petit et du plus petit au plus grand - et des comparaisons, fictives ou réelles ${ }^{3}$. Parce qu'il ouvre une nouvelle perspective, l'auteur du Monstre à explosion est, aux dires de Kundera, un véritable romancier : «(..) il ne recopiait pas des vérités brodées sur le rideau de la préinterprétation; il a eu le courage cervantesque de déchirer le rideau» (Kundera $2005: 149)^{4}$. Le romancier a vu quelque chose que tous pouvaient, ou auraient pu voir ou entendre, mais qu'ils n'avaient pas remarquée et dont ils avaient encore moins saisi la portée. Sa perspective a ainsi le potentiel de transformer celle de son lecteur.

Si j'aime l'histoire racontée par Kundera et l'expérimentation à laquelle il se prête avec son personnage, c'est qu'elles nous permettent de distinguer deux niveaux d'analyse : celui d'un homme, Monsieur Engelbert, et celui de l'observateur. Ce faisant, elles mettent en lumière un trait constitutif de ce que parvient à faire le «vrai» sociologue. Le premier niveau est celui du personnage, ce qu'il dit ou ce qu'il écrit dans son autobiographie. Bien que le bruit ennuie Monsieur Engelbert, il ne voit pas la portée du phénomène audelà de son existence personnelle ; il est, on le comprend, trop occupé à vivre sa vie, et à échapper au son des monstres à explosion. Le second niveau est

\footnotetext{
${ }^{2}$ Parmi les sociologues, Norbert Elias est un maître dans l'art du télescopage du petit au grand, de l'observation - des choses en surface, souvent les corps et les vêtements - et de la reconnaissance de l'importance et de l'emploi des romans (voir Kuzmics et Mozetic 2003 : 297 et suiv.). En littérature, la compréhension du nouveau monde prend notamment forme, chez Gombrowicz, dans la réaction pudique des cousines à la barbe rasée de l'oncle (2003 [1984] : 60); chez Broch (1990 [1931]), elle passe avant tout par le rapport qu'entretient l'officier Pasenow à son uniforme.

${ }^{3}$ Dans la recherche de relations de causalité, Max Weber procède par de multiples comparaisons, mais aussi par des "expérimentations de l'esprit» (Gedankenexperiment) qui s'apparentent à l'expérimentation de Kundera. Un autre des classiques de la sociologie, Georg Simmel, attire notre attention sur ceci : «Le comprendre relève davantage du créatif que du savoir à proprement parler ; les fragments, c'est-à-dire les faits positifs, dont se compose la vie des êtres humains, sont réunis en un tableau, produit de l'imagination sans être imaginaire » (Disselkamp 2012 : 155).

${ }^{4} L^{\prime}$ acte de déchirer le rideau de la pré-interprétation correspond à l'idée de rupture en sociologie.
} 
celui de l'observateur. Il voit ce que l'on n'aurait ni pu lire dans les journaux de l'époque ou dans l'ouvrage biographique d'Engelbert, ni non plus entendre dans les cafés de Prague. Il est à penser que ce qui paraît aux yeux d'Engelbert banal et futile, s'avère au contraire important pour l'observateur puisqu'il lui permet de " découvrir des liens là où l'on ignorait jusqu'alors qu'il y en eût ». Et c'est bien là, souligne le sociologue Norbert Elias, "la tâche centrale des recherches scientifiques » $(1991: 197)$.

L'histoire de Monsieur Engelbert illustre un trait constitutif du travail de sociologue. En distinguant le niveau des acteurs - des «personnages », dans le langage du roman — du niveau de l'observateur, elle permet de différencier un problème social d'un problème sociologique. Un problème social correspond à ce qui nous considérons important dans la vie de tous les jours. Il est fort probable que le personnage Engelbert considère digne d'intérêt, peu importe qu'il s'agisse de les dénigrer ou de les célébrer, le développement du jeune État et la montée de nouvelles élites ; la plupart d'entre nous accordons au moins un minimum d'intérêt à ce que nous lisons dans les journaux. Un problème sociologique est souvent lié à ces thèmes, mais ne s'y réduit pas ; dans une optique sociologique, les événements ne sont pas présentés à l'état brut. La sociologue reformule des questions, recadre et ouvre d'autres perspectives. Si elle n'est pas la seule à le faire - des romanciers, des critiques, des journalistes le font également (Mills 1967 [1959] : 19) —, elle a des outils qui lui sont propres: des théories qu'elle relit, des concepts qu'elle affine et des traditions qu'elle adapte. La perspective sociologique est également limitée par un objet empirique donné et sous-tendue par une question, l'observation, l'interprétation et, souvent, une volonté de synthèse.

\section{La sociologie comme mise en abîme}

Si l'exemple de Kundera m'a tout de suite plu, je n'ai pas immédiatement compris pourquoi. En y réfléchissant, j'ai réalisé qu'il s'agissait en fait d'une histoire dans une histoire : une mise en abîme, comme le disent les littéraires. C'est l'histoire de Kundera qui raconte l'histoire d'Engelbert dans le roman de John. La sociologie, à l'image de cet exemple, est toujours une mise en abîme. Elle fait toujours dialoguer plusieurs niveaux possibles d'interprétation pour recadrer une réalité. En ce sens, elle ne saurait être que reconstruction.

La sociologue opère une mise à distance, découpe la réalité qu'elle observe et les existences individuelles afin de les reconstruire, de les recomposer avec ses concepts et donc dans son langage (voir Simmel 1981 [1917] ; sur Weber, 
Colliot-Thélène 2006 : 41). Si ses points d'entrées et ses méthodes divergent de ceux du romancier, elle adopte une perspective analytique qui se distingue de celle des hommes et des femmes immergés dans une expérience quotidienne et immédiate. La prise en compte des différents niveaux en jeu met en lumière la capacité de détachement de l'observatrice et la rupture qu'elle opère, ce que Kundera appelle «déchirer le rideau $»^{5}$. Elle contribue à une plus grande réflexivité chez l'acteur ouvert à la perspective de la sociologue et, bien entendu, chez la sociologue elle-même.

Outre leur fonction de révélateur de la sociologie comme exercice de mise en abîme, l'exemple de Kundera et le détour par la littérature nous ouvrent deux autres pistes de réflexion : l'une sur cette époque à la fois contemporaine à Engelbert et à l'avènement de la sociologie, et l'autre sur ce genre particulier vers lequel Kundera choisit d'orienter les ambitions littéraires de son personnage, soit celui de l'ouvrage biographique.

\section{3 À L'intersection des deux perspectives}

\section{L'époque du roman et de la sociologie}

Le choix de ce premier quart du $20^{\mathrm{e}}$ siècle dans lequel John imagine se dérouler l'épisode de la vie d'Engelbert n'est pas anodin. Cette époque est celle d'étonnantes nouveautés politiques, socioéconomiques et techniques, une poussée réflexive et le début de l'ère de la sociologie.

Revenons au bruit. Ce thème est abordé par les sociologues comme par les romanciers. Pensons seulement au texte "Les grandes villes et la vie de l'esprit » du sociologue Georg Simmel (2004 [1903]) ou aux premières pages de L'Homme sans qualités du romancier Robert Musil (2004 [1930-1941]). Pourquoi cet intérêt pour des objets semblables à la même époque ? Kundera propose une réponse : «C'est précisément quand il était encore rare que le phénomène du bruit (bruit des moteurs) apparaissait dans toute son étonnante nouveauté. Déduisons-en une règle générale: la portée existentielle d'un phénomène social est perceptible avec la plus grande acuité non pas au moment de son expansion mais quand il se trouve à ses débuts, incomparablement plus faible qu'il ne le deviendra demain» (2005: 148). Ajoutons une deuxième règle à

\footnotetext{
${ }^{5}$ Cette faculté est d'autant plus perceptible si l'observateur écrit au «je », voir The Cop and the Sociologist (Thériault 2013a).
} 
celle énoncée par Kundera: les phénomènes sont plus perceptibles à leurs débuts, mais ils restent cependant à la surface de l'expérience de l'individu. En cela, j'entends qu'ils ne sont encore ni articulés ni détachés du « rideau de la préinterprétation ». Ils constituent en effet une masse informe d'irritations, d'énervements, d'enthousiasme, d'euphorie, de désirs et de jugements de valeur. Ils n'ont pas été «vus », cadrés, compris.

On doit probablement au roman la découverte de la réflexivité, cette nouvelle conscience et sensibilité qui fait l'objet de tant de romans de l'époque de l'auteur du Monstre à explosion et qui prend donne la mesure de la place de l'individu dans le monde, met en évidence la contingence et remet en question les hiérarchies sociales. Découverte par le roman, cette conscience a été aiguisée par la sociologie naissante. Ce n'est d'ailleurs pas un hasard si nombre de romans du premier tiers du $20^{\mathrm{e}}$ siècle - Thomas Mann, Hermann Broch, Robert Musil, Joseph Roth — ont stimulé l'imagination sociologique et sont toujours des sources d'inspiration pour notre discipline. Dès lors que la vision du roman et de la sociologie eût fait émerger une telle conscience, il n'y avait plus de retour en arrière : on voyait ce que l'on ne voyait pas auparavant. Cette prise de conscience avait le pouvoir de transformer la relation qu'on entretenait à la réalité.

\section{Et la question de la forme}

Kundera est conscient de l'importance de l'époque relatée dans Le Monstre à explosion et, volontairement ou non, il soulève une dernière question que j'aimerais aborder. S'il évoque l'exemple d'Engelbert pour illustrer la "spécificité du roman », il est notable qu'il le fasse par le biais de cette géniale petite expérimentation : sortir le personnage des pages du roman et lui confier la tâche d'écrire un ouvrage biographique.

La biographie était une forme littéraire très répandue en Europe dans le premier quart du 20 e siècle. Siegfried Kracauer [1889-1966], sociologue, historien, romancier et critique de cinéma contemporain de l'auteur du Monstre à explosion, décrit la biographie comme la forme de la «bourgeoisie stabilisée» (2008 [1930] : 84). Pour lui, la biographie constitue une forme appropriée à la bourgeoisie de l'époque : elle conforte l'investissement éthique et émotionnel que cette dernière place dans l'individu. Elle permet de prolonger l'existence d'une couche sociale dont la base s'est évaporée. Si la bourgeoisie est consciente $\mathrm{du}$ caractère anonyme qu'a pris l'individu au 
tournant du siècle, elle choisit la fuite et esquive la situation réelle tout en manifestant une volonté de salut (2008 [1930] : 85).

Cette tendance s'exprimerait-elle chez Engelbert ? Suivant la forme établie, il est à penser que le personnage condenserait dans son autobiographie l'histoire de sa vie: ses souvenirs ne contiendraient vraisemblablement ni les confessions, ni les détails intimes d'un journal; il y reconstruirait plutôt l'époque de sa naissance, la place et la forme qu'a prises son éducation, sa fonction professionnelle, les institutions politiques qui l'encadrent - et il ne manquerait sans doute pas d'écrire quelques réflexions sur la place de l'empereur au sein de l'Autriche-Hongrie. À partir d'une histoire à « caractère objectif », il écrirait sur des choses qu'il jugerait — avec plusieurs de ses semblables - importantes, et érigerait, " en toute modestie », un monument à sa personne, celle d'un homme du monde d'hier.

La période décrite dans le Monstre à explosion et la naissance de la sociologie en tant que discipline sont marquées par la recherche de formes. Il s'agit là d'un grand souci des auteurs du début du $20^{\text {e }}$ siècle, souci qui s'est manifesté dans ce qu'on a appelé la «crise du roman». La biographie constitue une réponse, critiquée, à cette crise, au « besoin d'une forme littéraire légitime » (Kracauer 2008 [1930] : 83). Kundera dédaigne cette forme qui, bien qu'elle s'approche du roman, ne peut ni ne souhaite déchirer le rideau de la pré-interprétation et donc opérer la rupture qu'il juge constitutive du rôle de romancier. Bien qu'il ait mêlé sa voix au cœur des critiques, Kracauer écrira lui-même une biographie (1994 [1937]). Il s'agit cependant d'un ouvrage qui se penche sur un homme qui émergea «des profondeurs de la masse » (2008 [1930] : 85) et qui exerça un genre considéré " populaire », Jacques Offenbach. Dans ses livres sur le roman (1986, 1993, 2005), Kundera prend partie pour les romanciers contre les sociologues : il joue, par exemple, Kafka contre Weber sur la bureaucratie. Mais rappelons-nous : plusieurs des romanciers et de ceux que l'on appellera éventuellement sociologues écrivent et empruntent à une forme et une plate-forme communes, le «feuilleton » publié, à l'époque, dans les pages des mêmes journaux. Cette tradition issue d'Europe centrale marie observations minutieuses et reportage, sociologie et littérature. Elle adopte souvent une forme légère, théorique, mais sans formalisme et souvent 
ironique. Il semble temps de plaider pour un retour à une telle forme pour écrire et, dans un même mouvement, penser la sociologie ${ }^{6}$.

\section{Bibliographie}

Broch, H. (1990 [1931]). Les somnambules. Paris, Gallimard.

Colliot-Thélène, C. (2006). La sociologie de Max Weber. Paris, La Découverte.

Disselkamp, A. (2012). «Le secret et la connaissance interpersonnelle: un fondement original du lien social ». Sociologie et sociétés 44(2), p. 143-163.

Elias, N. (1991 [1970]). Qu'est-ce que la sociologie? La Tour d'Aigues, Éditions de l'Aube.

Gombrowicz, W. (2003 [1984]). Souvenirs de Pologne. Paris, Gallimard.

Kundera, M. (2005). Le rideau. Essai en sept parties. Paris, Gallimard, p. 146-150.

—. (1993). Les testaments trahis : essai. Paris, Gallimard.

—. (1986). L'art du roman. Paris, Gallimard.

Kuzmics, H. et G. Mozetic (2003). Literatur als Soziologie. Zum Verhältnis von literarischer und gesellschaftlicher Wirklichkeit. Konstanz, UVK.

Kracauer, S. (2008 [1930]). «La biographie — la forme d'art néo-bourgeoise ». L'ornement de la masse. Essais sur la modernité weimarienne. Paris, La Découverte, p. 82-86.

—. (1994 [1937]). Jacques Offenbach, ou, Le secret du Second Empire, Gallimard.

Mills, C. W. (1967 [1959]). L'imagination sociologique. Paris, Maspero.

Musil, R. (2004 [1930-1941]). L'Homme sans qualités. Paris, Seuil.

Simmel, G. (2004 [1903]). «Les grandes villes et la vie de l'esprit ». Philosophie de la modernité. Paris, Payot, p. 169-183.

—. (1981 [1917]). «Le domaine de la sociologie», dans Sociologie et épistémologie, Paris, Presses universitaires de France, p. 83-105.

Thériault, B. (2013a). The Cop and the Sociologist: Investigating Diversity in German Police Forces. Bielefeld, transcript.

—. (2013b). «Feuilleton: ouverture à la nouvelle section». Sociologie et sociétés 45(2), p. 323-325.

\footnotetext{
${ }^{6}$ Voir la nouvelle section intitulée «Feuilleton » de la revue Sociologie et sociétés et le court texte publié en ouverture (Thériault, 2013b).
} 